\title{
Clinical Effect and Adverse Reactions of Modified Electro- convulsive Therapy for Refractory Obsessive-Compulsive Disorder
}

\author{
SHU XIN BAI*, BO LAN, DE-FANG LIU ${ }^{1}$, RU PEI LIANG ${ }^{1}$, GUO YONG LIU, DE BIN WANG ${ }^{2}$, SUO YUAN, WEN JIE SUN \\ AND GUANG YU LI
}

Department of Psychiatric Rehabilitation, Cang Zhou People's Hospital, Cangzhou, Hebei 061000, China; ${ }^{1}$ Department of Psychiatric Rehabilitation, Hua bei shi you psychiatric Hospital, Cangzhou, Hebei 061000,China; ${ }^{2}$ Department of orthopedics, Cang Zhou People's Hospital, Cangzhou, Hebei 061000 China

Bai et al:: Effect of Modified Electroconvulsive Therapy for Refractory Obsessive-Compulsive Disorder

To explore the clinical effect and adverse reactions of modified electroconvulsive therapy for refractory obsessive-compulsive disorder. 76 patients with refractory obsessive-compulsive disorder admitted to our hospital from June 2019 to February 2021 were selected and randomly divide into two groups, with 38 cases in each group. The control group was treated with conventional drug treatment and the study group was treated with modified electroconvulsive therapy on the basis of the control group. Follow-up was given every $6 \mathrm{w}$ for a total of $12 \mathrm{w}$ and corresponding evaluation was given every follow-up. The total effective rate of the study group was $92.11 \%$, which was significantly higher than that of the control group $(78.95 \%)$ and the difference was statistically significant $(p<0.05)$. There was no significant difference in Yale-brown obsessive compulsive scale, Hamilton anxiety scale, Hamilton depression scale, Montreal cognitive assessment scale and Tang danlin scores between the two groups before treatment $(p>0.05)$. At the 6th and 12th $w$ after treatment, it increased with time compared with that before treatment, The scores of Yale-brown obsessive compulsive scale, Hamilton depression scale and Hamilton anxiety scale decreased gradually. The difference was statistically significant $(p<0.05)$. The scores of Yale-brown obsessive compulsive scale, Hamilton anxiety scale and Hamilton depression scale in the study group were significantly lower than those in the control group at the 6th and 12th $\mathrm{w}$. The difference was statistically significant $(p<0.05)$, while the Montreal cognitive assessment scale scores and Tang danlin scores of patients in the study group were significantly higher than those in the control group at the 6th and 12th $w$ after treatment than before treatment and the difference was statistically significant $(p<0.05)$. The incidence of near memory impairment in the study group was significantly higher than that in the control group $(p<0.05)$, but there was no significant difference in adverse reactions such as agitation, blurred vision, constipation and abnormal examination items $(p>0.05)$. Modified electroconvulsive therapy for refractory obsessive-compulsive disorder has good clinical effect, low adverse reactions and can effectively improve patient's anxiety, depression and obsessive-compulsive symptoms, and improve patient's quality of life and cognitive function. However, there is a high incidence of near memory impairment.

Key words: Modified electroconvulsive convulsion; refractory obsessive-compulsive disorder; clinical efficacy; adverse reactions

Obsessive-compulsive disorder refers to chronic recurrent neurosis characterized by obsessivecompulsive concept, obsessive-compulsive behavior and obsessive-compulsive impulse. It coexists with conscious self-compulsion and self-countercompulsion, which leads to anxiety and depression due to their conflict. Most patients can recognize the abnormality of obsessive-compulsive symptoms, but it cannot be solved. As a result, some programmed thinking and behaviors are commonly used to relieve pain, resulting in social function damage of patients. In serious cases, behaviors such as suicide and selfinjury may occur, causing serious damage to patient's work, interpersonal relationships and other functions ${ }^{[1]}$. Refractory obsessive-compulsive disorder refers to patients with obsessive-compulsive disorder whose

*Address for correspondence

E-mail: baishuxin2008@163.com 
course of disease is more than $5 \mathrm{y}$ and who have applied two sufficient and sufficient anti-obsessivecompulsive drugs during treatment are ineffective, which is called refractory obsessive-compulsive disorder ${ }^{[2]}$. The lifetime prevalence rate of obsessivecompulsive disorder is $2 \% \sim 3 \%$, which is 3 times that of schizophrenia. Clinically, serotonin reuptake inhibitors are used for treatment, but $20 \% \sim 30 \%$ of patients are ineffective or have poor response to drugs ${ }^{[3]}$. Therefore, many scholars have carried out relevant research on effective treatment measures for refractory obsessivecompulsive disorder. Electroconvulsive therapy is a rapid and efficient treatment method to control patients with mental symptoms. However, during treatment, patients have clear consciousness and suffer from physical and mental torture, which is also easy to lead to fracture, cardiovascular and cerebrovascular diseases ${ }^{[4]}$. The modified electroconvulsive therapy is based on the traditional treatment plan with anesthetic and muscle relaxation, which is fast and safe, but there are few research results in China. Therefore, the purpose of this study is to explore its therapeutic effect on patients with refractory obsessive-compulsive disorder, so as to provide theoretical basis for clinical treatment. The results are as follows.

\section{MATERIALS AND METHODS}

\section{General data}

76 patients with refractory obsessive-compulsive disorder admitted to our hospital from June 2019 to February 2021 were selected. They were divided into two groups with 38 cases in each group by random number table method. The control group was treated with conventional drug therapy, while the study group was treated with modified electroconvulsive therapy on the basis of the control group. Follow-up was given every $4 \mathrm{w}$ for $12 \mathrm{w}$. Corresponding evaluation was given at each follow-up. Among them, there were 20 males and 18 females in the control group, aged 29.45 $\pm 10.23 \mathrm{y}$ old and the course of disease was 5.23 $\pm 2.14 \mathrm{y}$. In the observation group, there were 19 males and 19 females, aged $30.15 \pm 9.22$ y old and the course of disease was $5.17 \pm 1.16 \mathrm{y}$. There was no significant difference in age, gender and course of disease between the two groups ( $\mathrm{p}>0.05)$, which was comparable.

\section{Inclusion and exclusion criteria}

Inclusion criteria- All patients met the diagnostic criteria of obsessive-compulsive disorder of International Classification of Diseases 10th Edition (ICD-10) ${ }^{[5]}$.
Patients aged between 18 and $60 \mathrm{y}$ old, patients with the course of disease more than $5 \mathrm{y}$ and two kinds of anti obsessive compulsive drugs with different mechanisms of action used during the treatment. The sufficient amount and sufficient course of treatment were ineffective, patients with Yale-Brown Obsessive Compulsive Scale (Y-BCOS) score $\geq 20$. The study was approved by the hospital ethics committee, the patients and their families signed informed consent forms.

Exclusion criteria- Patients with severe loss or pathological changes of heart, liver, kidney and other organs, lactating or pregnant women, patients suffering from organic diseases of body and brain, patients with obsessive-compulsive disorder caused by secondary mental diseases, patients with speech communication barriers or different communicators.

\section{Treatment}

Both groups were given drug withdrawal and placebo cleaning $7 \mathrm{~d}$ before the start of the study. The control group was treated with clomipramine combined with olanzapine tablets. The initial dose of clomipramine was $25 \mathrm{mg}$, which was taken before going to bed. The dose was adjusted to $100 \mathrm{mg} 200 \mathrm{mg}$ per day within $10 \mathrm{~d}$. The olanzapine tablets were taken orally $2 \sim 3$ tablets per day, each $5 \mathrm{mg}$, for $12 \mathrm{w}$. Other anti-compulsive drugs and anti-psychotic drugs were stopped during the treatment. The study group was given modified electroconvulsive therapy on the basis of the control group. The specific operation is to give intravenous atropine $5 \mathrm{mg}$ before treatment, Then etomidate fat emulsion injection $0.3 \mathrm{mg} / \mathrm{kg}$ was injected, followed by intravenous injection of muscle relaxant succinylcholine chloride injection $2 \mathrm{mg} / \mathrm{kg}$ after anesthesia and then the patient was given bilateral temporal stimulation method, that is the stimulation electrode was placed on the temporal side of the patient and then the patient was given muscle tremor for $3 \mathrm{~min}$. After stimulation, the mask capsule was given for pressure artificial respiration until the patient's breathing recovered. The treatment was done once every other day, 2 3 times a week according to the patient's condition, for a total of $12 \mathrm{w}$.

\section{Observation index}

The clinical efficacy was evaluated by YB-OCS score. Before treatment and $12 \mathrm{w}$ after treatment, the curative effect was judged. Among them, the recovery rate of YB-OCS was $\geq 75 \%$, the markedly effective rate of YB-OCS was between $50 \%$ and $74 \%$, the effective 
rate of YB-OCS was between $25 \%$ and $49 \%$ and the ineffective rate of YB-OCS was less than $25 \%$.

The patients with obsessive-compulsive disorder were given YB-OCS scores before treatment, $6 \mathrm{w}$ and $12 \mathrm{w}$ of treatment. Hamilton Anxiety Scale ${ }^{[7]}$ Hamilton Anxiety Scale (HAMA) and Hamilton Depression Scale ${ }^{[8]}$ Hamilton Depression Scale (HAMD) were used to evaluate obsessive-compulsive symptoms and anxiety and depression. The YB-OCS score has both obsessivecompulsive thinking and obsessive-compulsive behavior, all of which adopt a 5-level scoring system of 0-4 points. Higher the score more will be the obvious obsessive-compulsive symptoms. HAMA and HAMD scores are $\leq 7$ as no anxiety or depression symptoms. $7 \sim 13$ points refer to the patient may have anxiety or depression. 14 20 points make it clear that patients have anxiety or depression. The score of $21 \sim 28$ is that the patient has obvious anxiety or depression. $\geq 29$ points refer to severe anxiety and depression.

The quality of life is assessed by Tang Danlin (TDL) Quality of Life Scale ${ }^{[9]}$, with 16 main items, including physical, psychological, social, due diligence and selfhealth awareness. The score is scored on a 5-point system. The choice is 5 points, generally 4 points, uncertainty 3 points, unlike 2 points, not 1 point. Higher the score, better the quality of life of the patient.

Cognitive function was evaluated by Montreal Cognitive Assessment Scale (MOCA), which involved abstract thinking, attention/concentration, language, executive function, memory, orientation, visual structure skills, etc., with a total of 11 items, with a full score of 30 . Higher the score, better the cognitive function of the patient.

The adverse reactions of the two groups were counted. The adverse reactions included near memory disorder, agitation, blurred vision, constipation, fidgety, insomnia, dry mouth, dizziness and headache, etc. Before and after treatment, the patients were given blood routine, renal function, liver function and electrocardiogram to evaluate the safety.

Follow-up was given every $6 \mathrm{w}$ for a total of $12 \mathrm{w}$ and corresponding evaluation was given every follow-up.

\section{Statistical analysis}

Data processing was carried out by SPSS 20.0 statistical software. The comparison of counting data between the two groups was expressed as the number of cases or percentage and chi-square test was used. The comparison of measurement data between the two groups was expressed as standard deviation \pm mean and $t$ test was used. The difference was statistically significant with $\mathrm{p}<0.05$.

\section{RESULTS AND DISCUSSION}

The total effective rate of the study group was $92.11 \%$, which was significantly higher than that of the control group $(78.95 \%)$, and the difference was statistically significant $(\mathrm{P}<0.05)$, as shown in Table 1 .

There was no significant difference in YB-OCS score, HAMA score and HAMD score between the two groups before treatment $(\mathrm{p}>0.05)$. However, at the 6th and 12th $\mathrm{w}$ after treatment, the YB-OCS score, HAMD and HAMA scores showed a gradual downward trend with the increase of time compared with those before treatment, with statistical significance $(p<0.05)$ and the YB-OCS score, HAMA and HAMD scores of patients in the study group were significantly lower than those in the control group at the 6th and 12th w, with statistical significance $(\mathrm{p}<0.05)$, as shown in Table 2 .

There was no significant difference in MOCA score and TDL score between the two groups before treatment ( $>0.05$ ), but the MOCA score and TDL score increased gradually with time at the 6th and 12th w after treatment compared with those before treatment, with statistical significance $(\mathrm{p}<0.05)$ and the MOCA score and TDL score of the study group were significantly higher than those of the control group at the 6th and 12th w with statistical significance $(\mathrm{p}<0.05)$, as shown in Table 3 .

The incidence of near memory disorder in the study group was significantly higher than that in the control group, with statistical significance $(\mathrm{p}<0.05)$, while there was no statistical significance in adverse reactions such as agitation, blurred vision, constipation and abnormal examination items ( $\mathrm{p}>0.05)$, as shown in Table 4 .

TABLE 1: COMPARISON OF CLINICAL EFFICACY BETWEEN THE TWO GROUPS

\begin{tabular}{lcccc}
\hline Curative effect & Study group $(\mathrm{n}=\mathbf{3 8})$ & Control group $(\mathrm{n}=38)$ & $\mathrm{X}^{2}$ & $\mathrm{p}$ value \\
\hline Recovered & 12 & 8 & 4.312 & 0.012 \\
Effective & 15 & 12 & 5.331 & 0.034 \\
Valid & 8 & 10 & 4.536 & 0.005 \\
Invalid & 3 & 8 & 7.245 & 0.007 \\
Total effective rate & $92.11 \%$ & 78.95 & 6.117 & 0.013 \\
\hline
\end{tabular}


TABLE 2: IMPROVEMENT OF OBSESSIVE-COMPULSIVE DISORDER AND ANXIETY AND DEPRESSION IN TWO GROUPS

\begin{tabular}{|c|c|c|c|c|c|}
\hline Scoring & & $\begin{array}{c}\text { Study group } \\
(n=38)\end{array}$ & $\begin{array}{c}\text { Control group } \\
(n=38)\end{array}$ & $\mathbf{t}$ & $p$ value \\
\hline & Before treatment & $25.94 \pm 4.17$ & $26.53 \pm 5.32$ & 0.146 & 0.613 \\
\hline \multirow[t]{3}{*}{ YB-OCS score } & 6 weeks after treatment & $16.23 \pm 4.76$ & $18.19 \pm 4.33$ & 6.412 & 0.012 \\
\hline & 12 weeks after treatment & $8.45 \pm 3.24$ & $9.57 \pm 3.16$ & 5.145 & 0.007 \\
\hline & Before treatment & $21.65 \pm 5.52$ & $21.83 \pm 4.22$ & 0.175 & 0.531 \\
\hline \multirow[t]{3}{*}{ HAMA score } & 6 weeks after treatment & $13.35 \pm 2.74$ & $15.65 \pm 3.17$ & 5.412 & 0.007 \\
\hline & 12 weeks after treatment & $6.72 \pm 2.53$ & $7.98 \pm 2.16$ & 6.114 & 0.013 \\
\hline & Before treatment & $21.18 \pm 5.44$ & $21.16 \pm 4.32$ & 0.432 & 0.578 \\
\hline \multirow[t]{2}{*}{ HAMD score } & 6 weeks after treatment & $12.35 \pm 3.24$ & $16.53 \pm 2.17$ & 5.316 & 0.025 \\
\hline & 12 weeks after treatment & $6.52 \pm 4.33$ & $7.85 \pm 3.16$ & 6.114 & 0.012 \\
\hline
\end{tabular}

TABLE 3: QUALITY OF LIFE AND COGNITIVE FUNCTION LEVEL OF PATIENTS IN BOTH GROUPS

\begin{tabular}{|c|c|c|c|c|c|}
\hline Score & & $\begin{array}{l}\text { Study group } \\
(n=38)\end{array}$ & $\begin{array}{c}\text { Control group } \\
(n=38)\end{array}$ & $\mathbf{t}$ & $p$ value \\
\hline \multirow{3}{*}{ MOCA score } & Before treatment & $19.54 \pm 1.32$ & $20.15 \pm 1.07$ & 0.537 & 0.746 \\
\hline & 6 weeks after treatment & $25.53 \pm 1.92$ & $22.54 \pm 1.31$ & 5.146 & 0.013 \\
\hline & 12 weeks after treatment & $27.78 \pm 1.46$ & $23.14 \pm 1.37$ & 6.713 & 0.011 \\
\hline \multirow{3}{*}{ TDL score } & Before treatment & $63.82 \pm 5.52$ & $65.53 \pm 4.19$ & 0.746 & 0.775 \\
\hline & 6 weeks after treatment & $70.26 \pm 14.37$ & $67.74 \pm 5.37$ & 5.413 & 0.014 \\
\hline & 12 weeks after treatment & $76.85 \pm 12.37$ & $72.31 \pm 4.23$ & 6.539 & 0.023 \\
\hline
\end{tabular}

TABLE 4: COMPARISON OF ADVERSE REACTIONS BETWEEN THE TWO GROUPS

\begin{tabular}{|c|c|c|c|c|c|}
\hline Group & & Study group $(n=38)$ & $\begin{array}{c}\text { Control group } \\
(n=38)\end{array}$ & $\mathrm{X}^{2}$ & $p$ value \\
\hline \multirow{8}{*}{$\begin{array}{l}\text { Adverse } \\
\text { reactions }\end{array}$} & Near memory disorder & 10 & 2 & 5.314 & 0.013 \\
\hline & Aggressive & 4 & 3 & 0.312 & 0.547 \\
\hline & Blurred vision & 8 & 5 & 0.642 & 0.113 \\
\hline & Constipation & 4 & 5 & 0.746 & 0.454 \\
\hline & Agitated sitting or standing & 5 & 3 & 0.852 & 0.139 \\
\hline & Insomnia & 1 & 2 & 0.846 & 0.237 \\
\hline & Dry mouth & 6 & 4 & 0.995 & 0.154 \\
\hline & Dizziness and headache & 8 & 7 & 0.743 & 0.617 \\
\hline \multirow{4}{*}{ Test Items } & Abnormal blood routine & 6 & 4 & 0.845 & 0.443 \\
\hline & Abnormal liver function & 5 & 3 & 0.255 & 0.647 \\
\hline & Abnormal renal function & 4 & 2 & 0.117 & 0.894 \\
\hline & $\begin{array}{c}\text { Abnormal electrocardiogram } \\
\text { examination }\end{array}$ & 4 & 3 & 0.832 & 0.097 \\
\hline
\end{tabular}

Obsessive-compulsive disorder has become a serious problem affecting human physical and mental health and public health. At present, researchers at home and abroad classify obsessive-compulsive disorder as the fourth common mental disease after depression, alcohol dependence and phobia. However, pre-illness personality, psychological factors, genetics and other aspects can all play a role in the occurrence of obsessivecompulsive disorder ${ }^{[11]}$. At present, there are research results on the mechanism of this disease, indicating that it may be related to dopamine dysfunction and 5-hydroxytryptamine (5-HT) hypofunction in the brain. Therefore, most patients often use selective 5-HT reuptake inhibitors to reduce the sensitivity of HT autoreceptors on the presynaptic membrane of 5-HT neuron terminals, which further inhibit the reuptake of 5-HT by neurons to improve the function of 5-HT and play a role in treating obsessive-compulsive disorder. However, there are still about $20 \% \sim 40 \%$ of patients who cannot be improved after sufficient and full course of treatment, thus becoming refractory obsessivecompulsive disorder ${ }^{[12]}$.

Non-convulsive electroconvulsive therapy is a commonly used physical therapy method for psychiatric diseases. It mainly stimulates the patient's brain through a short and appropriate amount of current under the condition of general anesthesia and muscle relaxation, resulting in extensive Electroencephalogram (EEG) attacks in the cerebral cortex, resulting in physiological 
and biochemical changes, and then treats the patient ${ }^{[13]}$. The results of this study show that the total effective rate of the study group is significantly higher than that of the control group, the YB-OCS score, HAMA score and HAMD score of the two groups decreased gradually with the increase of treatment time and the decrease degree of the study group was higher than that of the control group, suggesting that the application of modified electroconvulsive therapy (MECT) can effectively improve the clinical symptoms and anxiety and depression of patients with refractory obsessive-compulsive disorder. Meng Dexuan et al. ${ }^{[14]}$ research results show that fluvoxamine combined with MECT combined with small doses $(2.5,5.0 \mathrm{mg} / \mathrm{d})$ aripiprazole is effective and safe in the treatment of obsessive-compulsive disorder. The research results of Perugi et al. ${ }^{[15]}$ show that electroconvulsive therapy for severe and drug-resistant bipolar disorder is an effective and safe treatment method at all stages. Positive reactions were observed in about two thirds of cases and $80 \%$ of catatonic patients. The duration of the current attack is the main predictor of non-response. In fact, there is no risk of mania induced by electroconvulsive convulsion, and emotional instability is highly unlikely. For most severe patients with depression, mixed state, mania and tension, the application of electroconvulsive therapy can be effectively treated. The above results are consistent with the results of this study to a certain extent, which proves that the application of modified electroconvulsive therapy can effectively treat obsessive-compulsive disorder. In terms of treatment mechanism, some animal experiments show that electroconvulsive therapy on rats can increase the level of neurotrophic factors in brain regions of rats and cause nerve fibers to extend and germinate, resulting in neuron remodeling and regeneration, thus producing anti-obsessive-compulsive effect ${ }^{[16]}$. There are also research results that the application of MECS can cause stress response of hypothalamus, i.e. hypothalamuspituitary-adrenal cortex system, thus affecting the level of related neurotransmitters in patients, thus improving neurological function ${ }^{[17]}$. There are also research results showing that the effect of electroconvulsive therapy without convulsion is similar to that of 5-HT reuptake inhibitor to improve obsessive-compulsive disorder symptoms $^{[18]}$.

At present, a large number of research results show that obsessive-compulsive disorder patients have different degrees of cognitive impairment such as easy, attention and executive functions and their conversion ability is impaired ${ }^{[19]}$. Some research results show that the cognitive impairment of obsessive-compulsive disorder may be due to the impairment of prefrontal lobe function in obsessive-compulsive disorder patients, and the neural mechanism of cognitive function is controlled by the prefrontal lobe of the brain ${ }^{[20]}$. There are a large number of imaging tests showing focal abnormalities in frontal lobe, striatum and thalamus in patients with initial obsessive-compulsive disorder ${ }^{[21]}$. The results of this study showed that the MOCA score and TDL score of the two groups were significantly different before treatment. However, at the 6th and 12 th $\mathrm{w}$ after treatment, it increased with time compared with that before treatment. The MOCA score and TDL score gradually increased and the MOCA score and TDL score of the patients in the study group were significantly higher than those in the control group at the 6th and 12th w. The incidence of near memory impairment in the study group was significantly higher than that in the control group, suggesting that MECT for patients with refractory obsessive-compulsive disorder can effectively improve the cognitive function level of patients and improve the quality of life of patients. Gu Zhiwen et al. ${ }^{[22]}$ research results show that ketamine combined with propofol anesthesia MECT treatment of TRD patients can significantly improve clinical efficacy, improve cognitive function, serum BDNF level and serum BDNF/pro-BDNF ratio, reduce the incidence of EEG slow waves and have higher safety. Zhao Xuan et al. ${ }^{[23]}$ Research results show that lithium carbonate combined with electroconvulsive therapy can improve the depression and mania of patients with childhood traumatic bipolar disorder, and improve cognitive function and social behavior ability. The above results are consistent with the results of this study. All confirmed that for mental diseases, MECT can effectively improve the cognitive function and quality of life of patients. The specific improvement mechanism may be related to the increase of brain-derived neurotrophic factor stimulated by electroconvulsive stimulation, further promotes the remodeling and regeneration of related nerve fibers and neurons, further improves the level of cognitive function ${ }^{[24]}$, but there are also research results that electroconvulsive convulsion can cause damage to the short-term memory of patients, but can better restore the orientation of patients ${ }^{[25]}$. However, the recovery of cognitive function in this study may also be related to the basic drug treatment. In short, the mechanism of electroconvulsive therapy on cognitive function of patients with obsessive-compulsive disorder needs further exploration. 
To sum up the application of modified electroconvulsive therapy for refractory obsessive compulsive disorder has good clinical effect and low adverse reactions and can effectively improve anxiety, depression and obsessive-compulsive symptoms of patients, and improve the quality of life and cognitive function level of patients, but there is a defect of high incidence of near memory disorder.

\section{Acknowledgements:}

This work was supported by Cang Zhou People's Hospital and Hua bei shi you psychiatric Hospital, SHU-XIN BAI and BO LAN are contributed equally to this work, SHU-XIN BAI and BO LAN are considered co-corresponding in this paper.

\section{Conflicts of interest:}

The authors report no conflicts of interest.

\section{REFERENCES}

1. Richter PMA, Ramos RT. Obsessive-Compulsive Disorder. Continuum (Minneap Minn) 2018;24(3):828-44.

2. Robbins TW, Vaghi MM, Banca P. Obsessive-compulsive disorder: puzzles and prospects. Neuron 2019;102(1):27-47.

3. Figee M, Pattij T, Willuhn I, Luigjes J, van den Brink W, Goudriaan A, et al. Compulsivity in obsessive-compulsive disorder and addictions. Eur Neuro Psychopharmacol 2016;26(5):856-68.

4. Arya S, Filkowski MM, Nanda P, Sheth SA. Deep brain stimulation for obsessive-compulsive disorder. Bull Menninger Clin 2019;83(1):84-96.

5. Kempker AJ, Rudd KE, Wang HE, Martin GS. Sepsis Epidemiology Across the International Classification of Diseases, to International Classification of Diseases, Chasm A Direct Application of the Institute for Health Metrics and Evaluation Case Definition to Hospital Discharge Data. Critic Care Med 2020;48(12):1881-4.

6. Fatori D, Costa DL, Asbahr FR, Ferrão YA, Rosário MC, Miguel EC, et al. Is it time to change the gold standard of obsessive-compulsive disorder severity assessment? Factor structure of the Yale-Brown Obsessive-Compulsive Scale. Aus N Z J Psychiatry 2020;54(7):732-42.

7. Zimmerman M, Martin J, Clark H, McGonigal P, Harris L, Holst CG. Measuring anxiety in depressed patients: A comparison of the Hamilton anxiety rating scale and the DSM-5 Anxious Distress Specifier Interview. J Psychiatric Res 2017;93:59-63.

8. Çoban A, Tan O. Attention Deficit Hyperactivity Disorder, Impulsivity, Anxiety, and Depression Symptoms Mediating the Relationship Between Childhood Trauma and Symptoms Severity of Obsessive-Compulsive Disorder. Arch Neuropsychiatry 2020;57(1):37.

9. Weixia X, Ying L, Mingjun D. Clinical observation of antidepressants combined with short-term group cognitive behavioral therapy in the treatment of obsessive-compulsive disorder. Int J Psychiatry 2019;46(4):663-8.

10. Ciesielska N, Sokołowski R, Mazur E, Podhorecka M, Polak-Szabela A, Kędziora-Kornatowska K. Is the Montreal Cognitive Assessment (MoCA) test better suited than the Mini-Mental State Examination (MMSE) in mild cognitive impairment (MCI) detection among people aged over 60? Meta-analysis. Psychiatr Pol 2016;50(5):1039-52.

11. Bhikram T, Abi-Jaoude E, Sandor P. OCD: obsessivecompulsive disgust? The role of disgust in obsessivecompulsive disorder. J Psychiatry Neurosci 2017;42(5):300-6.

12. Skapinakis P, Caldwell DM, Hollingworth W, Bryden P, Fineberg NA, Salkovskis $\mathrm{P}$, et al. Pharmacological and psychotherapeutic interventions for management of obsessivecompulsive disorder in adults: a systematic review and network meta-analysis. Lancet Psychiatry 2016;3(8):730-9.

13. Kaliora SC, Zervas IM, Papadimitriou GN. Electroconvulsive therapy: 80 years of use in psychiatry. World J Psychiatry 2018;29(4):291-302.

14. CS Shastry. Efficacy and safety evaluation of fluvoxamine combined with different doses of aripiprazole in the treatment of obsessive-compulsive disorder. Anhui Med 2019;23(2):37982.

15. Perugi G, Medda P, Toni C, Mariani MG, Socci C, Mauri M. The role of electroconvulsive therapy (ECT) in bipolar disorder: effectiveness in 522 patients with bipolar depression, mixedstate, mania and catatonic features. Curr Neuropharmacol 2017;15(3):359-71.

16. Pusalkar M, Ghosh S, Jaggar M, Husain BF, Galande S, Vaidya VA. Acute and chronic electroconvulsive seizures (ecs) differentially regulate the expression of epigenetic machinery in the adult rat hippocampus. Int $\mathrm{J}$ Neuropsychopharmacol 2016;19(9):040.

17. Denysenko L, Sica N, Penders TM, Philbrick KL, Walker A, Shaffer S, et al. Catatonia in the medically ill: Etiology, diagnosis, and treatment. The Academy of ConsultationLiaison Psychiatry Evidence-Based Medicine Subcommittee Monograph. Ann Clin psychiatry 2018;30(2):140-55.

18. Tupal S, Faingold CL. Fenfluramine, a serotonin-releasing drug, prevents seizure-induced respiratory arrest and is anticonvulsant in the DBA/1 mouse model of SUDEP. Epilepsia 2019;60(3):485-94.

19. Benzina N, Mallet L, Burguière E, N'diaye K, Pelissolo A. Cognitive dysfunction in obsessive-compulsive disorder. Curr Psychiatry Rep 2016;18(9):1-8.

20. Hai Y, Xiaozhen N, Junjing Z. Study on the relationship between clinical characteristics and cognitive function of obsessive-compulsive disorder. J Med Forum 2019;40(1):76-7.

21. Zhang ZF, Fan Q, Bai YL. Magnetic resonance spectroscopy study on prefrontal aminobutyric acid level in patients with obsessive-compulsive disorder without medication. Shanghai Psychiatry 2016;28(5):263-70.

22. Zhiwen G, Xiong H, Chunping Z. Effect of ketamine combined with propofol anesthesia on the efficacy and cognitive function of modified electroconvulsive therapy for refractory depression. J Difficult Diseases 2021;20(1):47-52.

23. Xuan Z, Dong Z, Jia Z. Effect of lithium carbonate combined with non-convulsive electroconvulsive therapy on cognitive 
function and social behavior of childhood traumatic bipolar disorder. Chin Pharma 2020;29(20):67-9.

24. Qian C, Jiabo H, Chaoyun Z. The level of glial cell line-derived neurotrophic factor and cognitive function in refractory obsessive-compulsive disorder. Chin J Neuropsychiatry 2015;(8):497-500.

25. Hong Z, Bin Y, Guoling Z. Effect of electroconvulsive therapy on short-term memory impairment and recovery cycle in patients with schizophrenia. Shaanxi Med J 2018;47(2):162-4.
This is an open access article distributed under the terms of the Creative Commons Attribution-NonCommercial-ShareAlike 3.0 License, which allows others to remix, tweak, and build upon the work non-commercially, as long as the author is credited and the new creations are licensed under the identical terms

This article was originally published in a special issue, "Evolutionary Strategies in Biomedical Research and Pharmaceutical Sciences" Indian J Pharm Sci 2021:83(3)

Spl issue;120-126 